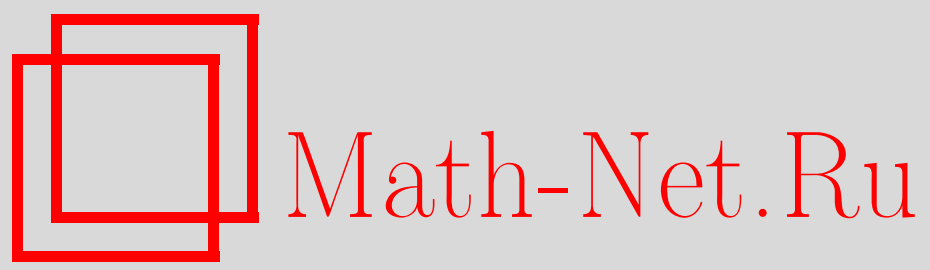

С. Волчёнков, Чем круг отличается от квадрата?, Квант, 2019, номер 4, 23-26

DOI: https://doi.org/10.4213/kvant20190403

Использование Общероссийского математического портала Math-Net.Ru подразумевает, что вы прочитали и согласны с пользовательским соглашением http://www.mathnet.ru/rus/agreement

Параметры загрузки:

IP: 18.208 .226 .222

26 апреля 2023 г., 12:29:08

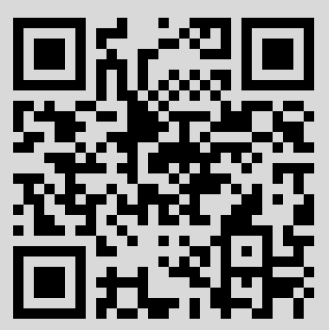




\section{Чем круг отличается от квадрата?}

\section{С.ВОЛЧЁНКОВ}

\section{ПЕРВОГО ВЗГЛЯДА ВОПРОС КАЖЕТ-}

ся странным. Каждый знает, и что такое круг, и что такое квадрат. И каждый знает, что это разные геометрические объекты. Но в этой статье речь пойдет не столько о геометрии, сколько о комбинаторной геометрии, где геометрические объекты рассматриваются с комбинаторной точки зрения - как элементы конечных множеств. Мы разберем три задачи, в которых круги и квадраты какими-то своими, на первый взгляд незначительными, различиями порождают различные ответы.

Задача 1 про круги. На плоскости расположены 5 кругов, каждые два из которых имеют общую точку. Верно ли, что всегда найдутся 3 круга, имеюших общую точку?

Ответ: верно.

Решение. Обозначим круги (и их центры) буквами $\mathrm{O}_{1}, \mathrm{O}_{2}, \mathrm{O}_{3}, \mathrm{O}_{4}, \mathrm{O}_{5}$ соответственно. Соединим центры друг с другом отрезками. Какие-то два из проведенных отрезков должны пересечься. Это известный факт (даже если точки пытаться соединять ломаными) - попробуйте доказать его самостоятельно или прочитайте в Приложении в конце статьи. Пусть отрезки $\mathrm{O}_{1} \mathrm{O}_{2}$ и $\mathrm{O}_{3} \mathrm{O}_{4}$ пересекаются в точке $X$ (рис.1). Осталось доказать, что верна такая лемма.

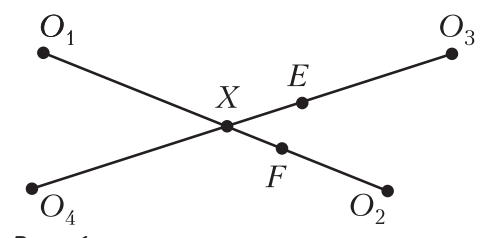

Pnc. 1

Лемма. Если есть две пары пересекаюшихся кругов ( пара $\mathrm{O}_{1}, \mathrm{O}_{2}$ и пара $\mathrm{O}_{3}$, $\mathrm{O}_{4}$ ), причем отрезки, соединяюшие их

DOI: https://doi.org/10.4213/kvant20190403 центры $\left(\mathrm{O}_{1} \mathrm{O}_{2}\right.$ и $\left.\mathrm{O}_{3} \mathrm{O}_{4}\right)$, имеют общую точку (X), то какие-то три из этих четырех кругов имеют общую точку.

Так как круги $O_{1}$ и $O_{2}$ пересекаются, на отрезке $\mathrm{O}_{1} \mathrm{O}_{2}$ есть точка $F$, принадлежащая им обоим. Аналогично, на отрезке $\mathrm{O}_{3} \mathrm{O}_{4}$ есть точка $E$, принадлежащая кругам $O_{3}$ и $O_{4}$. Пусть они расположены, как на рисунке 1 , причем $X E \geq X F$ (остальные случаи аналогичны). Тогда $\mathrm{O}_{4} E \geq \mathrm{O}_{4} X+X F \geq O_{4} F$, т.е. точка $F$ принадлежит кругам $\mathrm{O}_{1}, \mathrm{O}_{2}$ и $\mathrm{O}_{4}$. Лемма доказана.

Задача 1 про квадраты. На плоскости расположены 5 квадратов, каждые два из которых имеют общую точку. Верно ли, что всегда найдутся 3 квадрата, имеющих общую точку?

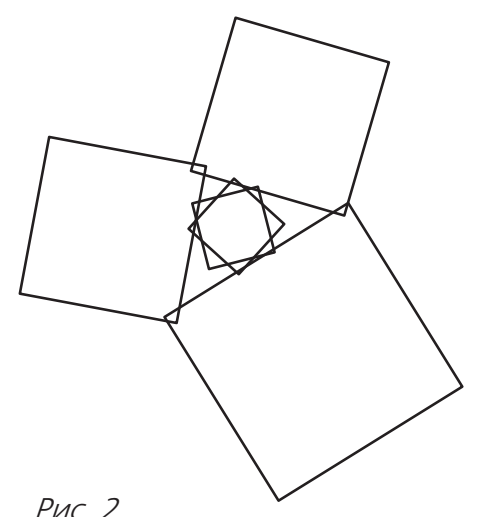

Ответ: неверно. Контрпример изображен на рисунке 2.

Задача 2 про круги. На плоскости расположены 3 красных и 3 синих круга, причем каждые два разночветных круга имеют общую точку. Верно ли, что всегда найдутся два одноцветных круга, имеющих общую точку?

Ответ: верно.

Решение. Соединим центры разноцветных кругов друг с другом отрезками. Какие-то два из проведенных отрезков долж- 
ны пересечься. Это тоже известный факт, часто называемый «три дома, три колодца» (докажите его самостоятельно или прочитайте в Приложении).Тогда, по лемме из задачи 1 , какие-то три круга имеют общую точку. А среди этих трех найдутся два круга одного цвета, что и требовалось.

Задача 2 про квадраты. На плоскости расположены 3 красных и 3 синих квадрата, причем каждые два разночветных квадрата имеют общую точку. Верно ли, что всегда найдутся два одноиветных квадрата, имеющих общую точку?

Ответ: неверно. Контрпример изображен на рисунке 3.

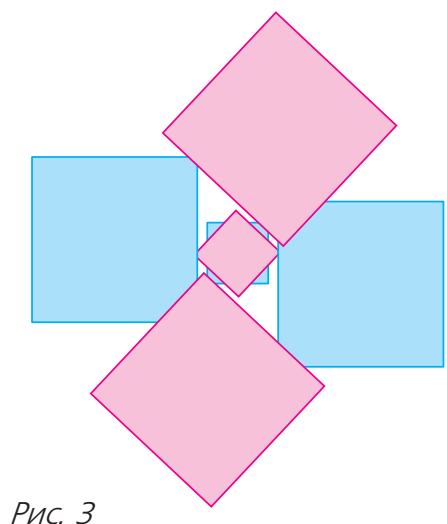

Замечание. В задачах 1 и 2 квадраты можно заменить на другие многоугольники, в частности на правильные $N$-угольники, где $N$ сколь угодно велико. При этом многоугольники становятся почти неотличимыми от кругов. Правда, рисунки 2 и 3 пришлось бы рассматривать под микроскопом, чтобы увидеть на них все пересечения.

Задача 3 про круги. $B$ выпуклом многоугольнике расположены $N$ непересекаюшихся кругов. Верно ли, что многоугольник всегда можно разрезать на $N$ выпуклых многоугольников, каждый из которых будет содержать ровно один из кру208?

Прежде чем переходить к решению этой задачи, рассмотрим более простую задачу .

Задача 3 про точки. В выпуклом многоугольнике отмечены $N$ точек ( с номерами от 1 до N). Верно ли, что многоугольник всегда можно разрезать на $N$ выпуклых многоугольников, каждый из которых будет содержать ровно одну отмеченную точку?

Ответ: верно.

Решение. Разобьем точки многоугольника на области с номерами от 1 до $N$ по такому правилу: область с номером $k$ состоит из точек, более близких к точке $k$, чем к любой другой отмеченной точке (такое разбиение называется диаграммой Вороного). При таком разбиении область с номером $k$ ограничена серединными перпендикулярами отрезков, проведенных из точки $k$ к остальным отмеченным точкам, т.е. является выпуклым многоугольником. Пример такой диаграммы приведен на рисунке 4 .

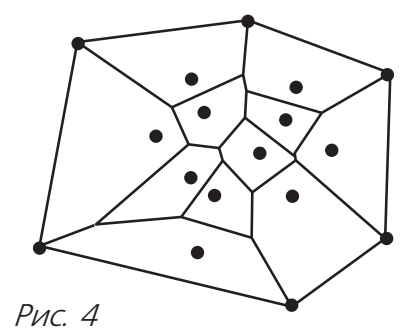

А теперь вернемся к задаче 3 про круги. Оказывается, что нужное нам разбиение многоугольника может быть образовано не серединными перпендикулярами, а так называемыми радикальными осями окружностей.

Радикальная ось двух непересекающихся окружностей - это геометрическое место точек, касательные из которых, проведенные к двум данным окружностям, имеют равные длины. Нетрудно показать, что радикальная ось двух окружностей любых радиусов с несовпадающими центрами это прямая (см. Приложение).

Если окружности не пересекаются, они будут целиком по разные стороны от радикальной оси. Разобьем тогда точки многоугольника (лежащие снаружи окружностей) на области, состоящие из точек, касательные из которых к одной окружности короче, чем к другим. Аналогично получим, что каждая окружность лежит целиком в одной области и эта область выпуклый многоугольник. 
Кстати, в случае «окружностей» нулевого радиуса касательные превращаются в расстояния до центров, и получается предыдущая задача.

И последняя задача.

Задача 3 про квадраты. $B$ выпуклом многоугольнике расположень $N$ непересекающихся квадратов. Верно ли, что многоугольник всегда можно разрезать на $N$ выпуклых многоугольников, каждый из которых будет содержать ровно один из квадратов?

Ответ: неверно. Контрпример изображен на рисунке 5.

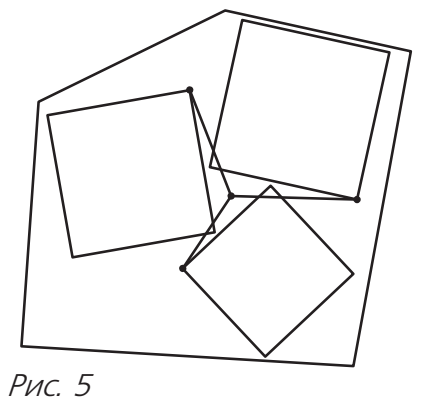

Пояснение. В пятиугольнике расположены три квадрата. Разрезать пятиугольник на три выпуклые части, каждая из которых содержит один квадрат, невозможно. Точка в центре рисунка не может принадлежать ни одной из частей, так как каждый из трех отрезков, идущих из нее к одной из точек квадратов, будет пересекать контур другого квадрата, т.е. ни один из этих отрезков не будет принадлежать выпуклой фигуре.

\section{Приложение}

Пять домов. Докажем, что на плоскости нельзя соединить друг с другом пять домов непересекающимися дорожками так, чтобы каждый дом был соединен с каждым отдельной дорожкой.

Допустим, это все-таки удалось сделать. Уберем все дорожки и будем возвращать их последовательно. Сначала добавим четыре дорожки, соединяющие четыре дома по циклу, получится «четырехугольник» из дорожек. Соединим теперь пары противоположных вершин этого четырехугольника (проведем «диагонали»). Одна диа- гональ будет внутри, другая - снаружи (рис. 6). В результате плоскость разделится на три «треугольника» и бесконечную

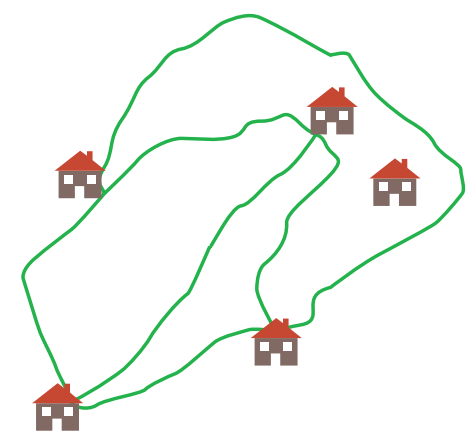

Pис. 6

часть, граница которой тоже будет треугольником (назовем эту часть «бесконечным» треугольником). Пятый дом окажется в одном из этих треугольников, и его можно будет соединить только с тремя вершинами этого треугольника. Противоречие - останется еще один дом, который нельзя соединить с пятым.

Три дома и три колодца. Докажем, ито на плоскости нельзя соединить непересекающимися дорожками три дома с тремя колодиами так, чтобы каждый дом был соединен с каждым колодием отдельной дорожкой.

Допустим, это все-таки удалось сделать. Уберем все дорожки и будем возвращать их последовательно. Сначала обойдем по замкнутому циклу все дома и колодцы. Так как дорожки не пересекаются, это будет «шестиугольник» из дорожек, причем дома и колодцы в нем чередуются (рис. 7). Осталось провести еще три дорожки, соединяющие противоположные вершины шестиугольника. Хотя бы две из

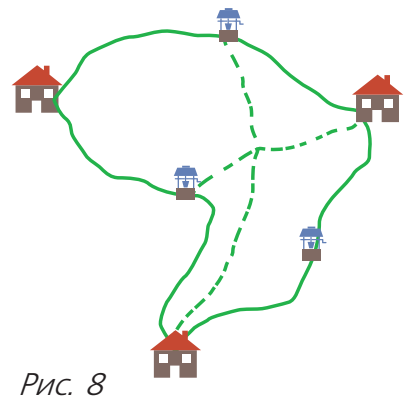

Pис. 8 
этих трех дорожек попадут обе либо внутрь, либо наружу шестиугольника и неизбежно пересекутся.

Радикальные оси. Пусть даны две окружности с различными центрами $\mathrm{O}_{1}, \mathrm{O}_{2}$ и радиусами $r_{1}, r_{2}$ соответственно (рис.8). Пусть точка $O_{1}$ имеет координаты $(a, b)$,

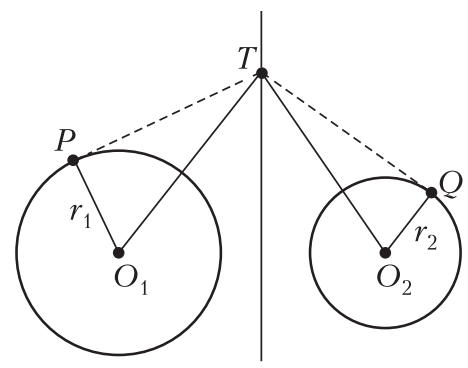

Pис. 8 а точка $O_{2}-$ координаты $(c, d)$. Найдем все такие точки $T(x, y)$, что касательные $T P$ и $T Q$ к нашим окружностям, проведенные из этой точки, равны. Касательные равны, когда равны их квадраты, а квадраты касательных можно найти по теореме Пифагора:

$T P^{2}=T O_{1}^{2}-O_{1} P^{2}=(x-a)^{2}+(y-b)^{2}-r_{1}^{2}$,

$T Q^{2}=T \mathrm{O}_{2}^{2}-\mathrm{O}_{2} Q^{2}=(x-c)^{2}+(y-d)^{2}-r_{2}^{2}$.

Приравнивая $T P^{2}$ и $T Q^{2}$, видим, что квадраты иксов и игреков взаимно уничтожаются и получается уравнение вида $m x+$ $+n y-k=0$, которое задает прямую, если хотя бы один из коэффициентов $m$ и $n$ ненулевой (а это так, поскольку центры окружностей различны).

\section{ЛАБОРА ТОРИЯ «К В АН ТА »}

\section{Опыт \\ по Галилею}

\section{М.СТАРШОВ}

ЕЛИКИЙ УЧЕНЫЙ СЕМНАДЦАТОГО ВЕ-

ка Галилео Галилей оставил богатое наследство в науке, занимаясь разнообразными исследованиями. Чаще всего вспоминают его работы по механике, оптике и астрономии. Гораздо реже отмечают вклад ученого в материаловедение, или в сопротивление материалов, по современной терминологии.

«Ни одна область физики не была столь тесно связана с потребностями практики, как физика твердого тела, т.е. физика тех материалов, которые применялись в строительном деле и в машиностроении...

Первым и важнейшим ответом на эту общественную потребность явилось произведение Галилея «Беседы и математические доказательства, касающиеся двух новых отраслей науки», написанное пленником инквизиции в 1638 г. в его загородном имении Арчетри и пересланное за границу через французского посла графа де Ноайль, <бывшего когда-то студентом Галилея в Падуе M.C.>. Две отрасли науки, о которых упоминается в заглавии, - это наука о движении... и наука о сопротивлении материалов. Этой второй отрасли науки фактически не было до Галилея». ${ }^{1}$

Галилей недоумевает, как это железный или стеклянный стержень выдерживает подвешенный к его концу значительный груз «в тысячу фунтов» (или в четыреста привычных для нас килограммов), но сломается от груза всего лишь в 50 фунтов, если конец стержня закреплен в стене, а груз прикреплен к другому его концу.

Дорфман видит у Галилея важные предположения, касающиеся вопроса о сопротивлении тел сгибанию, но непривычная терминология и сложные выводы освобождают его от рассмотрения всей проблемы. В самом деле, читать переводы великого итальянца очень тяжело, гораздо увлекательнее обсудить или даже провести некоторые эксперименты, отталкиваясь от идей Галилея.

1 Я.Г.Дорфман. Всемирная история физики (с древнейших времен до конца XVIII века). М.: Наука, 1974, с.214. 